\title{
Multilevel Polygonal Descriptor Matching Defined By Combining Discrete Lines And Force Histogram Concepts
}

\author{
L. Wendling ${ }^{1 *}$, I. Debled-Rennesson ${ }^{2}$, \\ H. Nasser ${ }^{2}$
}

the date of receipt and acceptance should be inserted later

\begin{abstract}
A new method allowing to describe shapes from a set of polygonal curves using a relational descriptor is proposed in this paper. An approach based on discrete lines at several increasing widths is run on the contour of an object to provide a multi-level polygonal representation from accurate description to more and more rough aspects. On each polygon is calculated a force histogram to define a relational feature signature following a set of directions integrating both spatial relation organization and disparities of the shape in a same distribution. Three different matching schemes are proposed to compare multilevel distributions: global representation, level to level following extracted maxima. This new method is fast and a first experimental study achieved on a common database shows its good behavior.
\end{abstract}

\section{Keywords}

Discrete lines, force histogram, polygonal representation, multilevel structure

\section{INTRODUCTION}

Shape representation methods are usually split into main categories: region-based approaches or contour-based approaches [1-3]. The choice of one representation instead of another one generally relies on the application under consideration. We focus on contour and more specifically on its polygonal description, consisting in a finite chain of straight line segments closing in a loop. Such a representation is widely used in several research fields such as graphic symbol recognition [4-7] or Geographic Information System [8].

Segments often serves a common basis for both syntactic and structural approaches. Already in the $90 \mathrm{~s}$ a string-matching technique to the problem of recognizing and classifying polygons has been defined by Maes [9] but this method is limited when the polygonal approximation of the object is inconsistent. Another

\footnotetext{
${ }^{1}$ Université Paris Descartes, LIPADE, 45, rue des Saints-Pères, 75270 Paris, France:

${ }^{2}$ Université de Lorraine, LORIA, UMR 7503, Vandoeuvre-lès-Nancy, F-54506, France.

*Corresponding author: Laurent.Wendling@parisdescartes.fr
} 
works focus for instance on the definition of a complete system for architectural drawing analysis [10] or symbol spotting [11].

Main well-known drawbacks of polygonal approximation rely on sensitivity to the noise or to over-segmentation effect considering the raster contour. Highly concave curves tend also to provide a bad discontinuity behavior on the achieved polygonal representation.

Since the last decades, numerous works have been carried out to assess the polygonal contour best fitting a raster contour. For line extraction, the Hough transform [12] has been widely used. But, applications are reasonably limited in case of degraded images due to its high computational cost. However, robust vectorization algorithms exist in line drawing images as in engineering drawings [1315]. The use of such low-level primitives varies widely in accordance with the complexity of the considered object. Efficient methods were also defined to take into account noise and/or over-segmentation by refining the process at different scales [16-19], etc. Despite existing relevant approximation works finding the "best" polygon is always a challenge which highly depends on the application under consideration.

In other words, since polygonal contour descriptors are based on the boundary of a shape, they cannot capture the internal structure of a shape. Furthermore, these methods are generally not suited to handle disjoint shapes or shapes with holes because the boundary information is not available. Moreover, a common drawback is error-prone raster to vector conversion inducing loss of information, which may result in lower recognition rates. On the other hand, region-based methods are more suited to general applications. However, they are generally more computationally intense and most approaches need to normalize (centroid position, re-sampling) the image to process with affine properties. Such a normalization introduce errors, sensitivity to noise, and thus inaccuracy in the recognition process.

In this paper we consider a powerful aim consisting in describing the shape at several scales from width of discrete lines [20]. Considering several widths allows to limit noise effect and limit artifact while limiting to some extent the influence of over-segmentation according to the processed widths. It allows to go progressively from an accurate representation to a more rough one while highlighting the main directions of the parts of the shape. Then we combine such description with a feature descriptor able to efficiently handle with holes or split parts of an object while integrating in its structure both relational parts and spatial variations [21]. This method can be assumed as hybrid; it combines a structural description of shapes with a statistical shape descriptor.

The outline of the paper is as follows. First the main frameworks required to define this new method are recalled in Section 2, that is discrete lines, blurred segments, and force histogram. The multi-polygonal structure is described in Section 3 as well as three basic matching strategies allowing to handle these representations. An experimental study performed on two common databases is given in Section 4 to show the interest of the proposed approach. Finally perspectives are provided in the conclusion. 


\section{BACKGROUND}

\subsection{Discrete Lines}

The arithmetical definition of discrete lines [20] is used in our method to decompose a discrete shape contour into discrete line segments.

A discrete line $\mathcal{D}(a, b, \mu, \omega)$, whose main vector is $(b, a)$, lower bound $\mu$ and thickness $\omega$ (with $a, b, \mu$ and $\omega$ being integer such that $\operatorname{gcd}(a, b)=1$ ) is the set of integer points $(x, y)$ verifying $\mu \leq a x-b y<\mu+\omega$.

In this work, we study sequences of points, corresponding to shape contours, and we compute polygonal approximations containing all the points of the sequences.

More precisely, let us consider a sequence of points $\mathcal{S}_{b}$, it is named a blurred segment of width $\nu$ if there exists a discrete line $\mathcal{D}(a, b, \mu, \omega)$ containing all the points of $\mathcal{S}_{f}$ and such that $\frac{\omega-1}{\max (|a|,|b|)} \leq \nu$. This discrete line is called the optimal bounding line of $\mathcal{S}_{b}$.

A linear algorithm was proposed in [20] to incrementally obtain the characteristics of the optimal bounding line of a sequence of points. It relies on the linear and incremental computation of the convex hull of the scanned point sequence as well as on the arithmetical and geometrical properties of discrete lines.

Based on this algorithm, an interesting geometrical structure can be computed on a shape contour: the width- $\nu$ tangential cover. It consists in the sequence of all maximal blurred segments of width $\nu\left(M B S_{\nu}\right)$ located on the shape contour (see Fig. 1). The common zone of several $M B S_{\nu}$ can contain a point with high curvature, called dominant point. A method to detect dominant points from a width- $\nu$ tangential cover of a contour is proposed in $[22,23]$, a polygonal representation of the contour is then deduced. Different values of width then induce several polygonal simplifications of a same contour (see Fig. 4).
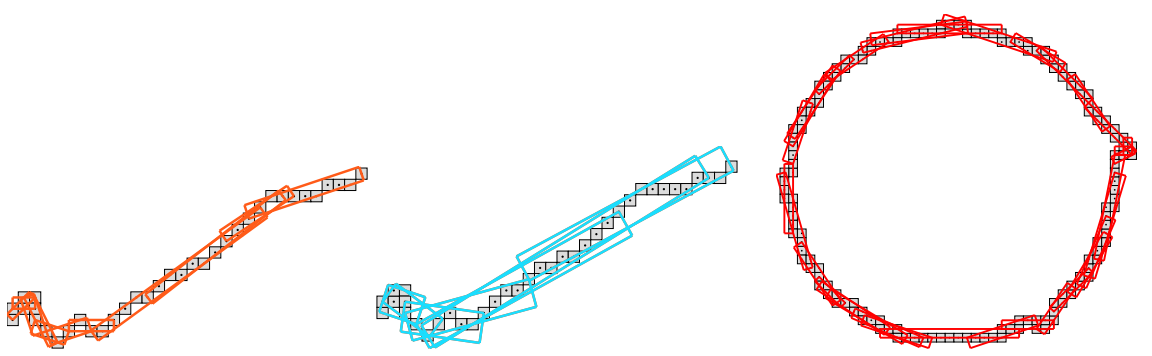

Fig. 1 On the left, the width-1 tangential cover of a piece of a shape contour. On the center, the width- 2 tangential cover of the same piece. On the right, the width-1 tangential cover of a noisy discrete circle. 


\subsection{Force Histogram}

\subsubsection{Force Histogram of a shape}

The histogram of forces was initially defined to assess the spatial relation between two binary objects but it can be easily used to provide a discriminate signature (and descriptor) characterizing one object. We recall here basic notions aiming at describing such a kind of signature which efficiently integrates both whole shape and spatial description following a set of directions. A full description of the underlying theoretical developments can be found in $[24,21,25,26]$.

The attraction force between two points at a distance $d$ is given by:

$$
\forall d \in R_{+}^{*}, \varphi_{r}(d)=1 / d^{r}
$$

with $r$, the kind of force processed, e.g. $r=0$ for constant forces, which are suitable considering one object and $r=2$ for gravitational ones.

The handling of segments is considered to decrease the computation time instead of directly studying any pair of points. Let $I_{1}$ and $I_{2}$ be two segments beared by a line of angle $\theta_{k}$ from the frame, $D_{I_{1} I_{2}}^{\theta_{k}}$ the distance between them and |.| the length of a segment. The calculation of the attraction force $f_{r}$ of a segment with regard to another is given by:

$$
f_{r}\left(\left|I_{1}\right|, D_{I_{1} I_{2}}^{\theta_{k}},\left|I_{2}\right|\right)=\int_{D_{I_{1} I_{2}}^{\theta_{k}}+\left|I_{2}\right|}^{\left|I_{1}\right|+D_{I_{1} I_{2}}^{\theta_{k}}+\left|I_{2}\right|} \int_{0}^{\left|I_{2}\right|} \varphi_{r}(u-v) d v d u
$$

Considering one raster object $A$, following a direction $\theta_{k}$ it can be entirely described by the set of segments beared by a pencil of parallel lines of angle $\theta_{k}$ from the orthogonal frame. Let us take one line, denoted $\mathcal{D}_{\eta}^{\theta_{k}}$. The set of $S$ segments corresponds to: $A_{\theta_{k}}(\eta)=\cup\left\{I_{i}\right\}_{i=1, S}$ and the mutual attraction of these segments is given by:

$$
F\left(\theta, A_{\theta_{k}}(\eta), A_{\theta_{k}}(\eta)\right)=\sum_{i \in 1 . . S} \sum_{j \in 1 . . S} f_{r}\left(\left|I_{i}\right|, D_{I_{i} I_{j}}^{\theta_{k}},\left|I_{j}\right|\right)
$$

Due to superimposed segments, constant forces are suitable in our study. An approximation of gravitational force impact might be done using points while avoiding superimposed points and linked points at a null distance. The last constraint can be eventually removed by using a sub-pixel modeling. Another way can be to introduce progressive forces (as in [24]) but this generally leads to minor the impact of gravitational ones considering compact shapes.

\subsubsection{Force histogram of a polygon}

Using raster data all the pencils of lines $\mathcal{D}_{\theta}^{\eta}$ which entirely describe $A$ are then processed and the associated mutual attraction summed. Considering a polygonal description of $A$, noted $P^{A}$, the calculation is based on a set of regular polygons having 3 or 4 vertices (see Fig. 2). All the lines $\mathcal{D}_{\theta}^{\eta}$ bearing a polygonal point are projected on a perpendicular straight line to set the intervals describing $A[24,21$, 26]. Then $A$ consists in a set of trapezium (or triangle) whose height is given by 


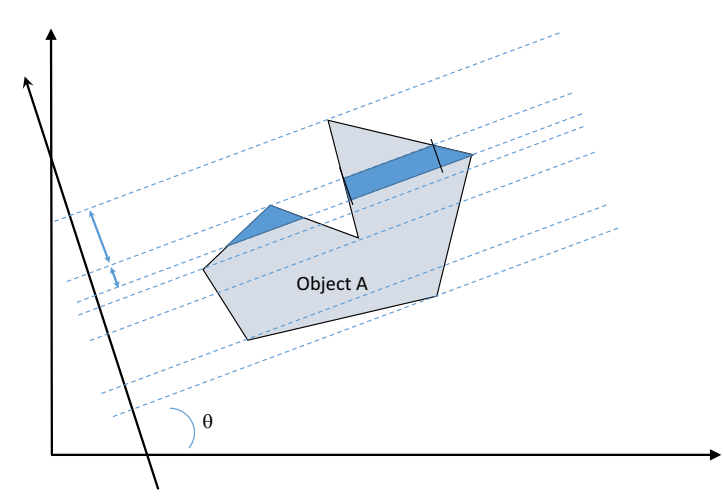

Fig. 2 Set of triangles or trapezium describing an object $A$ following a direction $\theta$.

the width of intervals, combined with bases to calculate the associated attraction and summed (Riemann sum).

The calculation of $F^{A A}(\theta)$, respectively $\mathcal{F}^{\mathcal{P}^{A}}(\theta)$, remains to an assessment of the forces exerted by an object with itself in the direction $\theta$.

Let us consider a set of $p$ directions $\Theta=\left\{\theta_{k}\right\}_{k=1, p \wedge \theta_{k} \in[-\pi,+\pi)}$ with a constant step. Finally the calculation of $F^{A A}$ onto $\Theta$ defines a spatial relational descriptor, denoted $\mathcal{F}^{A}$ for raster data and $\mathcal{F}^{P^{A}}$ for polygonal one. The calculation of the complexity depends on the sorting of the projected vertices. That is in $\mathcal{O}(p v \ln (v))$ with $v$ the number of vertices of the polygon.

\section{MULTI-POLYGONAL MATCHING}

\subsection{Multi-Polygonal Structure}

Let $A$ and $B$ be two shapes and let $\mathcal{P}^{A}=\cup_{\omega_{i} \in \Omega}\left(P^{A_{\omega_{i}}}\right)$ and $\mathcal{P}^{B}=\cup_{\omega_{i} \in \Omega}\left(P^{B_{\omega_{i}}}\right)$ be their multi-polygonal representation obtained using $n$ increasing width $\Omega=$ $\left\{\omega_{1}, \omega_{2} \ldots \omega_{n}\right\}$ with $\omega_{i}<\omega_{i+1}$ and $P^{A_{\omega_{i}}}$ the polygon calculated from the width $w_{i}$. The force histogram (or distribution) is calculated on each representation from the set of points defining each polygon following $p$ directions $\Theta$ (see previous section). Then the multi-polygonal descriptor associated to a shape $A$ is denoted by:

$$
\mathcal{F}^{\mathcal{P}^{A}}=\bigcup_{\omega_{i} \in \Omega}\left(\mathcal{F}^{P^{A \omega_{i}}}\right)
$$

\subsection{Multi-Polygonal Descriptor Properties}

By axiomatic definitions of the function $F$, the following properties can be checked and easily introduced in the matching process following the specificity of the application under consideration:

- Translation as polygon are processed independently of their location in an image. Only points and the whole shape of the polygon are considered. 
- Symmetry according to a mirror representation of the polygon. In this case processing the directions in the opposite direction allows to directly take into account the symmetry property.

- Homothety according to a normalized distribution. Considering real points, the global shape of the polygon is the same at different scales. Obviously considering discrete data, high disparities might occur at very low scales. However the whole representation of the force histogram remains stable in most of the cases due to the low sensitivity to noise of the approach $[21,25]$.

- Rotation because the approach is isotropic. A rotation of the polygon relies on circular shifts on the achieved force histogram. An assessment of the rotation (not invariant) can be carried out by minimizing the distance between two superimposed force histograms at different shifts.

Such properties rely on each polygonal representation $P^{A_{\omega_{i}}}$ but they can be easily extended to the whole description $\mathcal{F}^{\mathcal{P}^{A}}$ as they are calculated from the same located shape and so apply along a common principle at each associated level $\mathcal{F}^{P_{\omega_{i}}^{A}}$.

It is important to notice that if a shape contains several holes having or not parts of shapes implying a set of external and internal polygons, it is always possible to calculate the associated distribution due to the bi-linearity property. That is trapezium describing the holes are not integrated in the calculation of $F$. In other words, it is not useful to process holes during the matching step as they are directly taken into account inside of the force histogram.

\subsection{Matching Strategies}

Three schemes able to match two multi polygonal distributions (MPD) $\mathcal{F}^{\mathcal{P}^{A}}$ and $\mathcal{F}^{\mathcal{P}^{B}}$ are defined in this section. Let $\mathcal{M}$ be a metric, or a similarity ratio, calculating a score between two distributions.

\subsubsection{Weighted histogram matching}

The first scheme, denoted by $M_{1}$ relies on the calculation of a global distribution $\mathcal{G}\left(\mathcal{F}^{\mathcal{P}^{A}}\right)$ related to a shape $A$ by aggregating the distributions achieved at each level. Let $\theta_{k}$ be a direction, which corresponds to the $k^{\text {th }}$ bin of the distribution. A weighted scheme similar to [27] is used here as follows (assuming that $\omega_{0}=0$ ).

$$
\mathcal{G}\left(\mathcal{F}^{\mathcal{P}^{A}}\left(\theta_{k}\right)\right)=\sum_{i=1, n}\left(\omega_{i}-\omega_{i-1}\right) \mathcal{F}^{\mathcal{P}^{A \omega_{i}}}\left(\theta_{k}\right)
$$

And so on, considering all the $p$ directions (see section 2.2) to define the average distribution $\mathcal{G}\left(\mathcal{F}^{\mathcal{P}^{A}}\right)$. The distance $\Delta_{M_{1}}$ between two MPD $A$ and $B$ is directly given by:

$$
\Delta_{M_{1}}(A, B)=\mathcal{M}\left(\mathcal{G}\left(\mathcal{F}^{\mathcal{P}^{A}}\right), \mathcal{G}\left(\mathcal{F}^{\mathcal{P}^{B}}\right)\right)
$$


Considering raster data, a similar scheme was successively applied to classify chest radiograph image view but considering $\mathrm{F}$-signatures computed on a set of image binarisation [28]. Here the modeling is based on polygon handling and the weight is set from the difference between successive width values.

\subsubsection{Level polygonal feature set matching}

The second scheme, noted $M_{2}$, relies on an assessment level per level of both distributions which are weighted as in $M_{1}$ by the difference of two successive levels, that is:

$$
\Delta_{M_{2}}(A, B)=\frac{1}{n} \sum_{i=1, n}\left(\omega_{i}-\omega_{i-1}\right) \mathcal{M}\left(\mathcal{F}^{P^{A \omega_{i}}}, \mathcal{F}^{P^{B \omega_{i}}}\right)
$$

The interest of this scheme is to study the similarity of pairwise distributions at each level $\omega_{i}$. The aggregation is quite similar as the one of Krishnapuram et al. [29] who modeled standard relationships between fuzzy sets described by level cuts. However we do not ensure a convex hull representation and we put no hypothesis about polygonal level membership.

\subsubsection{Key direction matching}

At last, a third scheme $M_{3}$ is based on the matching of a set of meaningful direction values, denoted by $\Phi$, consisting in local minimum and maximum points extracted from the derivative of each distribution. Let us consider a distribution $A$ at width $\omega_{i}, \Phi_{\omega_{i}}^{A}$ corresponds to the set of directions corresponding to the loci of both extrema and minima of $\mathcal{F}^{\mathcal{P}^{A \omega_{i}}}$, that is:

$$
\begin{aligned}
\Phi_{\omega_{i}}^{A}=\left\{\theta_{k} \mid \theta_{k} \subset \Theta \backslash\left\{\theta_{1}, \theta_{p}\right\} \wedge\right. & \left(\mathcal{F}^{\mathcal{P}^{A \omega_{i}}}\left(\theta_{k-1}\right)<\mathcal{F}^{\mathcal{P}^{A \omega_{i}}}\left(\theta_{k}\right)>\mathcal{F}^{\mathcal{P}^{A \omega_{i}}}\left(\theta_{k+1}\right)\right. \\
& \left.\left.\vee \mathcal{F}^{\mathcal{P}^{A \omega_{i}}}\left(\theta_{k-1}\right)>\mathcal{F}^{\mathcal{P}^{A \omega_{i}}}\left(\theta_{k}\right)<\mathcal{F}^{\mathcal{P}^{A \omega_{i}}}\left(\theta_{k+1}\right)\right)\right\}
\end{aligned}
$$

Due to the circular representation (modulo the size $p$ ), $\theta_{1}$ and $\theta_{p}$ are separately processed. Considering two objects $A$ and $B$, the matching is similar to $M_{2}$ but limited to the set of suitable directions $\Phi_{\omega_{i}}^{A}$ and $\Phi_{\omega_{i}}^{B}$ extracted at each level $\omega_{i}$ :

$$
\Delta_{M_{3}}(A, B)=\frac{1}{n} \sum_{i=1, n}\left(\omega_{i}-\omega_{i-1}\right) \mathcal{M}\left(\mathcal{F}_{\Phi_{\omega_{i}}^{A} \cup \Phi_{\omega_{i}}^{A}}^{P_{\omega_{i}}^{B}}, \mathcal{F}_{\Phi_{\omega_{i}}^{A} \cup \Phi_{\omega_{i}}^{B}}^{P_{\omega_{i}}^{B}}\right)
$$

In some extent, the underlying idea of the well-known curvature scale space method [30] is followed. However the derivative does not require Gaussian filters to smooth the distribution in order to focus on key points. The computation of force histogram integrates the whole shape according to progressive directions and the studied distributions are most of the time regular with no high disparities. 


\section{EXPERIMENTAL STUDY}

4.1 Databases and metrics

Two well-known shape databases provided by Sharvit et al. [31] are used to test our methods. The first database $B 1$ consists in nine categories with 11 shapes in each cluster and the second one $B 2$ consists in 18 categories of 12 shapes. Considering $B 1$ a few of the shapes are occluded (airplanes and hands) and some shapes are partially represented (rabbits, men, and hands). There are also distorted objects (tools) and heterogeneous shapes in the same cluster (animals) (see Fig. 3). The database $B 2$ contained also shapes having similar disturbance.

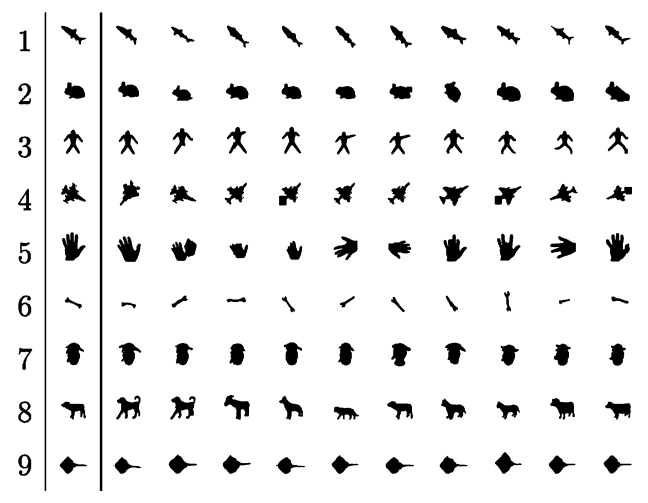

Fig. 3 Sharvit'dabatase composed of 99 shapes.

Such databases are widely used to compare feature descriptors which are more adapted to classify raster pattern due to noise, artifacts and distorted parts. For instance, the generic Fourier descriptor [3] provides high recognition rates on these databases. Here we consider only polygonal representations of shapes which are obviously more sensitive to noise effect and pattern distortions. The aim is to show the interest of such multi-polygonal structure through the matching strategies $M_{1}, M_{2}$ and $M_{3}$. Several metrics and similarity ratio $\Delta$ will be tested as Jaccard, Tanimoto, $C h i^{2}$ and Hellinger. Due to the weak number of samples the evaluation process is similar to a leave one out considering the distance of each sample with the remaining database (as $k=1$ ) to avoid a bias.

\subsection{Evaluation}

\subsubsection{Multi-scale structure}

We provide in Fig. 4 an example of polygonal decomposition following four widths to better visualize the structure used in this paper. Two samples belong to the same cluster (fish) and another belong to the cluster hand.

It may be noted that the small elements (polygonal contour in green in Fig. 4) of the structure are better preserved at low width (for instance the fins of the 


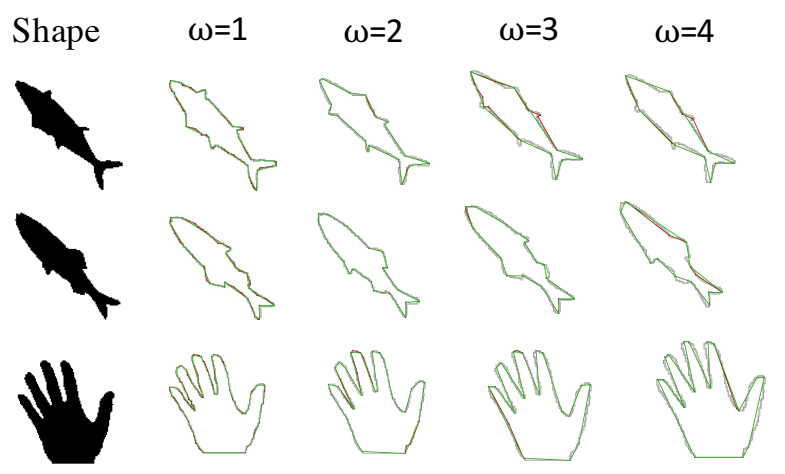

Fig. 4 Multi-Polgonal representation following width.

fishes) and a larger width concentrates rather meaningful directions of the shape (the palm of the hand is smoothed as well as fish fins).

Few shapes and the force histogram calculated on associated polygon defined from the width $\omega_{1}$ are given in Fig. 5 .

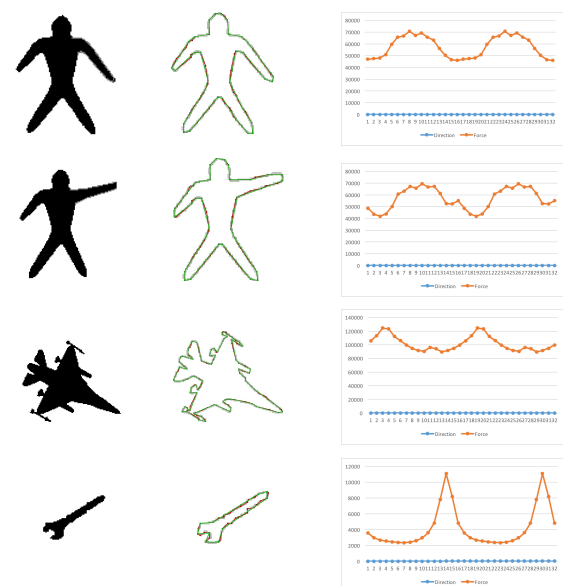

Fig. 5 Force histogram calculated on Polygons (width $\omega_{1}$ ).

The difference between the shapes can be directly seen in the distributions. For instance the arm raised induced a directional perturbation visible on the second force histogram. The last two force histogram samples indicate the main direction of the shape (plane and tool) while integrating the width of the objects (see the pick of the tool force histogram). 


\subsubsection{Number of directions}

Tests are done on Sharvit $B 1$ to study the impact of the underlying parameters. Five increasing widths are considered for the multi-polygonal representation of all the shapes. The influence of the number of directions $p$ is studied (see Table 1 ).

\begin{tabular}{|c|c|c|c|c|}
\hline Directions & 128 & 64 & 32 & 16 \\
\hline$M_{1}$ & 68.9 & 84.4 & $\mathbf{8 3 . 3}$ & $\mathbf{8 3 . 3}$ \\
\hline$M_{2}$ & 72.2 & 85.6 & 85.6 & $\mathbf{8 7 . 8}$ \\
\hline$M_{3}$ & 67.8 & 76.7 & $\mathbf{8 1 . 1}$ & 80.0 \\
\hline
\end{tabular}

Table 1 Recognition rates obtained using the Jaccard index.

The achieved results (using the Jaccard index) show a good behavior of the three methods except for a large number of directions. Many spatial directions introduce more irrelevant information during matching and thus reduce the impact of significant directions.

\subsubsection{Distance and Similarity}

Following the previous study, $p$ is set to 32 directions (16 is also a good compromise). Five usual similarity ratio and distance are studied (see Table 2). We also add a contour based approach $S_{a}$ which consider circular directions (here 360) defined from the centroid of the shape as in [32].

\begin{tabular}{|c|c|c|c|c|}
\hline Methods & $M_{1}$ & $M_{2}$ & $M_{3}$ & $S_{A}$ \\
\hline Jaccard & 83.3 & $\mathbf{8 5 . 6}$ & 81.1 & $\mathbf{7 4 . 4}$ \\
\hline Tanimoto & 83.3 & $\mathbf{8 5 . 6}$ & 81.1 & $\mathbf{7 4 . 4}$ \\
\hline$L_{1}$ & 82.2 & $\mathbf{8 5 . 6}$ & $\mathbf{8 2 . 2}$ & $\mathbf{7 4 . 4}$ \\
\hline Chi $^{2}$ & $\mathbf{8 4 . 4}$ & $\mathbf{8 5 . 6}$ & $\mathbf{8 2 . 2}$ & 73.3 \\
\hline Hellinger & $\mathbf{8 4 . 4}$ & $\mathbf{8 5 . 6}$ & $\mathbf{8 2 . 2}$ & 66.7 \\
\hline
\end{tabular}

Table 2 Recognition rates. Metrics and Similarity

The three methods are few sensitive to usual metrics (or similarity). This good behavior, as well as the good recognition rates, shows that the information stored in the force histogram distributions, which integrates both the organization of the spatial relationships and the disparities of the shape in the same distribution, are discriminating and robust at the metric.

\subsubsection{Increasing number of cuts}

The impact of increasing number of widths is considered (see Fig. 6). A step set at 0.5 between each width is considered. The number of directions $p$ is set to 16 and Tanimoto index is applied.

An increase in recognition rates is obtained for all three methods. The method $M_{1}$ is sensitive to the number of processed polygons. So, the weighted sum of force histogram becomes less discriminating when polygons of increasing width 


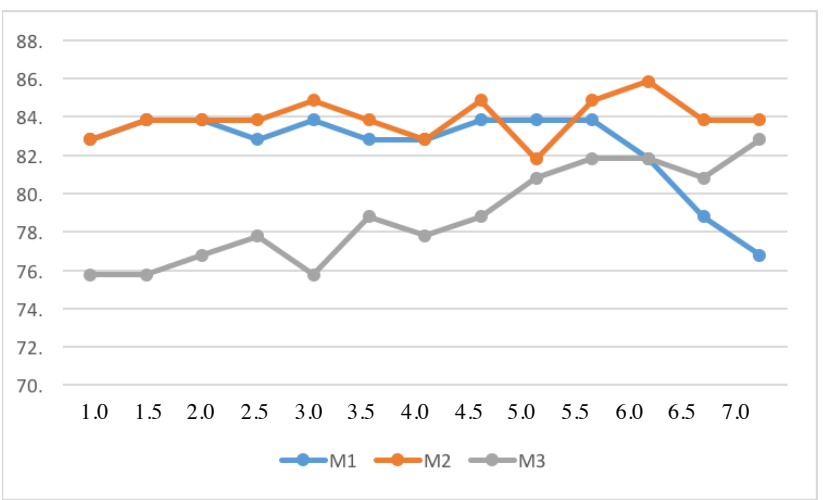

Fig. 6 Recognition rates. Increasing number of cuts.

are added. The method $M_{2}$ remains stable most of the time, showing the interest of a matching level per level, that is following the same width. The behavior of the method $M_{3}$ is interesting as the score increases gradually, showing the influence of main directions at high level.

\subsubsection{Test on Sharvit B2}

Another test is carried out on the database $B 2$. We consider values of parameters similar to those used to make tests on $B_{1}$, that is $p=32$, five widths and the Jaccard index. We also consider a polygonal representation method, denoted by $K M$, which provide a polygon close to the raster contour of a shape [17]. The force histogram is calculated on it to make a comparison.

\begin{tabular}{|c|c|c|c|c|c|}
\hline Methods & $M_{1}$ & $M_{2}$ & $M_{3}$ & $K M$ & $S_{A}$ \\
\hline Rate & 84.3 & $\mathbf{8 6 . 7}$ & 81.5 & 83.4 & 63.0 \\
\hline
\end{tabular}

Table 3 Recognition rates (32 bins - Jaccard Index)

The results are close to those ones obtained using $B 1$ despite an increasing number of samples showing the good behavior of our approaches. Handling with a multi-level approach seems to better take into account the main directional regions of a shape than a relevant method $K M$ where the polygonal contour fits well the pattern but only at one scale.

\subsection{Discussion}

Both methods $M_{1}$ and $M_{2}$ provide interesting results while having a robust behavior independently of the metric used.

Most of the recognition rates obtained with $M_{2}$ are better than those ones of $M_{1}$ showing the interest of handling the polygons, level per level, instead of averaging the amount of associated force histograms. 
Considering $M_{3}$ the results are a bit disappointing even few directions are processed (lower than $13 \%$ in average). This emphasizes that the relational curve between the key directions remains a discriminant information. Furthermore this method has an interesting behavior when the number of processed polygons increases.

\section{CONCLUSION}

Original methods aiming at representing a shape from a set of distributions associated to polygons have been proposed in this paper. These approaches combined two powerful concepts: discrete lines and force histogram. Achieved results on two databases are very promising. Furthermore the methods are fast and ensure a robust matching.

New developments integrating the interval between key directions or enabling to process with a different number of polygons per representation are under consideration. As the larger the value of the width, the rougher the aspect of the polygon is, further investigations are also dedicated to the search of the optimal description of each sample of a database.

\section{References}

1. S. Loncaric, "A survey of shape analysis techniques," Pattern Recognition, vol. 31, no. 8, pp. $983-1001,1998$.

2. A.K. Jain, R.P.W. Duin, and J. Maoa, "Statistical pattern recognition: a review," IEEE Transactions on Pattern Analysis and Machine Intelligence, vol. 22(1), pp. 4-37, 2000.

3. D. Zhang and G. Lu, "Shape-based image retrieval using generic fourier descriptor," Sig. Proc.: Image Comm., vol. 17, no. 10, pp. 825-848, 2002.

4. L.P. Cordella and M. Vento, "Symbol recognition in documents: a collection of techniques?," IJDAR, vol. 3, no. 2, pp. 73-88, 2000.

5. K. C. Santosh, Document Image Analysis - Current Trends and Challenges in Graphics Recognition, Springer, 2018.

6. H.S. Baird and K. Tombre, "The evolution of document image analysis," in Handbook of Document Image Processing and Recognition, pp. 63-71. 2014.

7. K.C. Santosh and L. Wendling, "Graphical symbol recognition," in Encyclopedia of Electrical and Electronics Engineering, Wiley, pp. 1-22. 2014.

8. M. Duckham, L. Kulik, M.F. Worboys, and A. Galton, "Efficient generation of simple polygons for characterizing the shape of a set of points in the plane," Pattern Recognition, vol. 41, no. 10, pp. 3224-3236, 2008.

9. M. Maes, "Polygonal shape recognition using string matching techniques," Pattern Recognition, vol. 24, no. 5, pp. 443-440, 1991.

10. P. Dosch, K. Tombre, C. Ah-Soon, and G. Masini, "A complete system for the analysis of architectural drawings," IJDAR, vol. 3, no. 2, pp. 102-116, 2000.

11. M. Rusiñol, J. Lladós, and G. Sánchez, "Symbol spotting in vectorized technical drawings through a lookup table of region strings," Pattern Anal. Appl., vol. 13, no. 3, pp. 321-331, 2010 .

12. Dana H. Ballard, "Generalizing the hough transform to detect arbitrary shapes," Pattern Recognition, vol. 13, no. 2, pp. 111-122, 1981

13. R.D.T. Janssen and A.M. Vossepoel, "Adaptive vectorization of line drawing images," Computer Vision and Image Understanding, vol. 65, no. 1, pp. 38-56, 1997.

14. X. Hilaire and K. Tombre, "Robust and accurate vectorization of line drawings," IEEE Trans. Pattern Anal. Mach. Intell., vol. 28, no. 6, pp. 890-904, 2006.

15. J. Song, F. Su, C.-L. Tai, , and S. Cai, "An object-oriented progressive-simplification based vectorization system for engineering drawings: Model, algorithm, and performance," IEEE Transactions on Pattern Analysis and Machine Intelligence, vol. 24(8), pp. 1048-1060, 2002. 
16. I. Debled-Rennesson, S. Tabbone, and L. Wendling, "Multiorder polygonal approximation of digital curves," Electronic Letters on Computer Vision and Image Analysis, vol. 5, no. 2, pp. 98-110, 1981.

17. K. Dilip and K.H.L. Maylor, "Polygonal representation of digital curves," in Digital Image Processing. InTech, 2012.

18. B. Kerautret and J.-O. Lachaud, "Meaningful scales detection along digital contours for unsupervised local noise estimation," IEEE Trans. Pattern Anal. Mach. Intell., vol. 34, no. 12, pp. 2379-2392, 2012.

19. A. Vacavant, T. Roussillon, B. Kerautret, and J.-O. Lachaud, "A combined multiscale/irregular algorithm for the vectorization of noisy digital contours," Computer Vision and Image Understanding, vol. 117, no. 4, pp. 438-450, 2013.

20. I. Debled-Rennesson, F. Feschet, and J. Rouyer-Degli, "Optimal blurred segments decomposition of noisy shapes in linear time," Computers 86 Graphics, vol. 30(1), pp. 30-36, 2006.

21. P. Matsakis and L. Wendling, "A new way to represent the relative position between areal objects," IEEE Transactions on Pattern Analysis and Machine Intelligence, vol. 21(7), pp. 634-643, 1999.

22. TP. Nguyen and I. Debled-Rennesson, "A discrete geometry approach for dominant point detection," Pattern Recognition, vol. 44, no. 1, pp. 32-44, 2011.

23. H. Nasser, P. Ngo, and I. Debled-Rennesson, "Dominant point detection based on discrete curve structure and applications," J. Comput. Syst. Sci., vol. 95, pp. 177-192, 2018.

24. P. Matsakis, "Relations spatiales structurelles et interprtation dimages," in PhD Thesis. Université Paul Sabatier, Toulouse, 1998.

25. S. Tabbone, L. Wendling, and K. Tombre, "Matching of graphical symbols in line-drawing images using angular signature information," IJDAR, vol. 6, no. 2, pp. 115-125, 2003.

26. P. Matsakis, L. Wendling, and J. Ni, "A general approach to the fuzzy modeling of spatial relationships," in Methods for Handling Imperfect Spatial Information, Methods for Handling Imperfect Spatial Information, Springer Ed. R. Jeansoulin, O. Papini, $H$. Prade, S. Schockaert, pp. 49-74. 2010.

27. D. Dubois and M.-C. Jaulent, "A general approach to parameter evaluation in fuzzy digital pictures," Pattern Recognition Letters, vol. 6(4), pp. 251-259, 1987.

28. K.C. Santosh and L. Wendling, "Angular relational signature-based chest radiograph image view classification," Med. Biol. Engineering and Computing, vol. 56, no. 8, pp. 1447-1458, 2018.

29. R. Krishnapuram, J.M. Keller, and Y. Ma, "Quantitative analysis of properties and spatial relations of fuzzy image regions," IEEE Trans. Fuzzy Systems, vol. 1, no. 3, pp. 222-233, 1993.

30. F. Mokhtarian, "Silhouette-based isolated object recognition through curvature scale space," IEEE Trans. Pattern Anal. Mach. Intell., vol. 17, no. 5, pp. 539-544, 1995.

31. D. Sharvit, J. Chan, H. Tek, and B. Kimia, "Symmetry-based indexing of image databases," J. Visual Communication and Image Representation, vol. 9, no. 4, pp. 366380, 1998.

32. T. Bernier and J.-A. Landry, "A new method for representing and matching shapes of natural objects," Pattern Recognition, vol. 36, no. 8, pp. 1711-1723, 2003. 